\title{
CONFIGURACIÓN BIOPOLITICA DE LAS NECESIDADES DEL CAMPESINO COLOMBIANO EN LOS AÑOS $90^{*}$
}

Fecha de entrega: 20171006

Fecha de evaluación: 20171027

Fecha de aprobación: 20171126

\author{
Luz Helena Di Giorgi Fonseca**
}

\section{Resumen}

El contexto rural colombiano en los años 90 se configuró a partir de un discurso de desarrollo que desconoció y estigmatizó a la población campesina. En este sentido, "el trabajador agrario", tal y como lo considera la Constitución Política de 1991, no refiere a la realidad específica de las comunidades campesinas, sino a un "pequeño productor", que como lo indica el Informe de Desarrollo Humano de 2011, representa a individuo con un rol secundario, que con asistencia crediticia y técnica puede ponerse a nivel, pero solo como productor de mediana escala. El presente artículo de reflexión tiene por objetivo analizar los efectos biopolíticos que ha tenido la visión política del desarrollo implementada en el campo colombiano en esta época,

* El artículo hace parte de la revisión teórica, vinculado a la investigación doctoral que realizar la autora, que se titula: "Análisis de las formas de agencia campesina conformadas con el paro nacional agrario y popular (PNAP) realizado en el 2013. Un acercamiento desde las representaciones simbólicoafectivas que se articulan y ponen en tensión con el ejercicio del derecho y la movilización social". Esta investigación se realiza para el doctorado en Derecho en la Universidad del Rosario. Citar como: Di Giorgi , L. (2018). Configuración biopolítica de las necesidades del campesino colombiano en los años 90. Cuadernos de Filosofia Latinoamericana, 39(118), 13-36. DOI: https://doi.org/10.15332/10.15332/ s0120-8462.2018.0118.01.

* Doctoranda de Derecho en la Universidad del Rosario, magister en Filosofía de la Universidad del Rosario y filósofa de la Universidad Libre. Actualmente es docente en la Universidad del Rosario para la Facultad de Jurisprudencia y Escuela de Ciencias Humanas. Correo electrónico: luz.digiorgi@urosario. edu.co, helenadigiorgi@gmail.com 
haciendo énfasis en la forma como se han establecido las necesidades para esta población.

Palabras clave: campesinos, biopolítica, representaciones simbólicas, agencia y necesidades.

\section{Abstract}

Since the decade of the nineties, the Colombian rural context has configured from a developmental discourse which started to be implemented for a farmer who at present, is not only unknown but also stigmatized by the institutions. In this sense, the "rural worker" as it is considered by the Colombian Constitution of 1991, does not refer to the specific reality of the rural communities, as indicated by the Report on Human Development from 2011, but to a "small producer" who represents to an individual with a secondary role with both technical and financial assistance that can be placed at the level of others, yet only as a producer of medium scale. The reflex article is aimed to analyze the bio-political effects which have had the political point of view of the present development introduced in the Colombian countryside emphasizing the way how the needs for this population have been managed.

Keywords: Farmers, biopolitics, symbolic representation, agency and needs.

\section{Resumo}

O contexto rural colombiano nos anos 90 configurou-se a partir de um discurso de desenvolvimento que desconheceu e estigmatizou a população camponesa. Neste sentido, "o trabalhador agrário", como o considera a Constituição Política de 1991, no relacionado à realidade especifica das comunidades camponesas, senão ao "pequeno produtor",

* $\quad 0$ artigo faz parte da revisão teórica, vinculado à pesquisa doutoral que realiza a autora, que se titula: "Análise das formas da agência camponesa conformadas com a greve nacional agrária e popular (PNAP) realizada em 2013. Uma aproximação desde as representações simbólico-afetivas que se articulam e tensionam com o exercício do direito e a mobilização social". Esta pesquisa realiza-se para o doutorado em Direito na Universidade do Rosário. 
que como indica o Informe de Desenvolvimento Humano de 2011, representa ao individuo com um rol secundário, que com ajuda creditícia e técnica pode se colocar ao nível, mas só como produtor de mediana escala. Este artigo de reflexão tem como objetivo analisar os efeitos biopolíticos que tem tido a visão política do desenvolvimento implementada no campo colombiano em aquela época, enfatizando na forma como tem se estabelecido as necessidades para esta população.

Palavras-chave: camponeses, biopolítica, representações simbólicas, agência e necessidades.

\section{Introducción}

El Informe de Desarrollo Humano del 2011 (PNUD) concluye que en Colombia hay una falla en el reconocimiento político y jurídico de la población campesina, pues los instrumentos que los incluyen (leyes, políticas públicas, planes desarrollo y herramientas de diagnóstico), los considera bajo nociones que no tienen nada que ver con esta comunidad, por el contrario, se refieren a ellos a partir de representaciones que provienen de discursos foráneos. Categorías tales como: "trabajador agrario" o "productor agropecuario", limitan y sesgan la comprensión del modo de ser del campesino. Dada esta constitución negativa del campesino colombiano, el artículo hace un análisis biopolítico del discurso del desarrollo, implementado para el contexto rural, en los años noventa, principalmente, indaga por los efectos que tienen estas representaciones en su reconocimiento, especialmente, en la constitución de una su identidad y agencia.

La hipótesis central del texto, radica en demostrar que gran parte de los saberes y estrategias que han sido implementadas, a través de las políticas que hacen referencia al contexto rural colombiano, por lo menos aquellas que provienen de la época del desarrollismo -años 90- no hablan propiamente de los problemas, ni de las necesidades que estas personas han demandado por periodos de tiempo (Salgado, 2014); por el contrario, tanto su identidad y necesidades han sido impuestas, a través de medidas de asistencia que incentivaron el establecimiento de alianzas empresariales, junto con el fortalecimiento de nuevas técnicas de producción, incluso fortaleciendo las formas tradicionales, con el fin de que los sectores campesinos se provean a sí mismos sus recursos -en casos en donde es favorable-, reduciendo así la responsabilidad del 
Estado, y por tanto, dejando cada vez la carga a individuos que no han sido reconocidos y que se encuentran en situación de desigualdad. En este sentido, las preguntas que el artículo aborda son las siguientes: ¿cómo opera el carácter biopolítico del discurso del desarrollo en el contexto rural?, ¿cuáles son las estrategias biopolíticas que revisten las políticas que configurar el contexto rural, promulgadas desde los años 90?, ¿cuáles han sido sus consecuencias? Para dar respuesta a estas preguntas, la primera parte del texto aborda la noción de biopolítica, junto con el papel que juega en la comprensión de la implementación del discurso del desarrollo en Colombia; la segunda parte hace un análisis del caso colombiano, específicamente una reflexión en torno a las representaciones simbólicas y sus efectos en la constitución de la identidad y las necesidades de los campesinos en los años 90; finalmente se hace una mención de las consecuencias en las formas de producción rural.

\section{Incursión de la vida a en la política}

La perspectiva biopolítica que consolida Michael Foucault $(2000,2006,2007)$ en sus cursos impartidos en el Collège de France entre los años 1976 y 1979, hacen referencia a las formas de control y administración de la vida, a partir de ciertos conocimientos científicos que empezaron a desarrollarse a finales del siglo XVIII y que tuvieron incidencia en el campo político. Este vínculo comienza a surgir justo cuando los distintos saberes (la biología, la antropología, las ciencias sociales, etc.) logran descubrimientos acerca de las leyes con las que opera la vida y su comportamiento. Si bien desde Aristóteles se concebía que los seres humanos están dispuestos a la política, ya en la época moderna es la política la que los toma por objeto. En sentido, muestra la forma como la política ejerce y tiene unos efectos en la vida cotidiana, no de manera vertical, sino a partir de situaciones horizontales de poder que parecen obvias e imperceptibles. Este nuevo rol que ocupa la vida en política, introduce la preocupación moderna de qué vidas deben ser preservadas y la manera sobre cómo deben preservarse. No obstante, esta implicación de la vida en la política, según Foucault, presenta por lo menos dos transformaciones importantes. En primer lugar, se estableció la anatomopolitica, que a partir de la consolidación del poder soberano moderno, con la consigna hacer morir o dejar vivir (siglos XVII y XVIII), se implementaron técnicas disciplinarias, que comenzaron a controlar y adiestrar al cuerpo individual, para hacerlo más dócil. 
La definición que Schmitt (2014) hace del poder soberano, radica en la decisión que fija por la necesidad de establecer la normalidad a través de la ley en una situación caótica. En este sentido, pareciera que en el momento en que se da una modificación al orden social, el derecho es irrealizable y se necesita de un estado de excepción que expida un nuevo derecho que se corresponda al nuevo orden social y jurídico acaecido. En este caso, hay que señalar que la decisión de la situación normal, según Schmitt (2012), supone un orden a priori. La excepcionalidad no solo se relaciona con la toma de una decisión desde un punto de vista político, sino jurídico, dado que esta se constituye en el momento en el que, por medio de un sistema de normas, se pretende reafirmar una normalidad concreta.

En el siglo XIX se evidencia la modificación de este modelo, ya que busca ejercer un control de la vida de forma distinta, pues en pro del desarrollo de la vida, hay un auge de estrategias políticas, bajo la consigna de un hacer vivir y dejar morir. A este tipo de poder soberano lo llama biopolítica de la población, que refiere a técnicas en donde no se ejerce el poder sobre el cuerpo individual, sino sobre la vida en su conjunto, sobre la población y la especie, en este caso, la vida comienza a verse sometida a análisis estadísticos, relacionados con la natalidad, la reproducibilidad, la longevidad y la mortalidad, con el fin de normalizar la vida, pues "el conjunto de mecanismos por medio de los cuales aquello que en la especie humana constituye sus rasgos biológicos fundamentales, podrá ser parte de una estrategia de poder" (Foucault, 2007, p. 15). Este aspecto es importante para el desarrollo de procesos económicos capitalistas, ya que hace posible la inserción de los cuerpos a los métodos productivos, pues estas técnicas son "capaces de aumentar las fuerzas, las aptitudes y la vida general, sin por ello tornarlas más difíciles de dominar" (Foucault, 2007, p. 170). La categoría de población comienza a cobrar importancia, en tanto que hace referencia a un sujeto constituido por un conjunto de individuos, que por medio de ciertos mecanismos se pretende que este se conduzca de un modo determinado (Foucault, 2006), a diferencia de la noción de pueblo, que para la época era concebido como las gentes que hacían resistencia a la determinación social.

Existen por lo menos dos elementos que actualmente amplían la reflexión en biopolítica, en primer lugar, la idea de inmunidad que sugiere Roberto Espósito (2006), en segundo lugar, la focopolítica que propone Sonia Álvarez (2002, 2005), dicho término es una nueva cara de la biopolítica, que incentiva formas de autocontrol y autogestión individual y comunitaria, específica o sectorizada, que se materializa 
por medio de políticas sociales que junto a organismos internacionales, promueven un nuevo humanitarismo.

Por un lado, Espósito (2006) introduce el concepto de inmunidad, que consiste en el establecimiento de decisiones políticas, que ligadas a un conocimiento científico, tienen la intención de proteger la vida de aquello que la contamina. Este sentido, debido a la protección de un tipo de vida particular, se es capaz de negar la vida misma, al respecto afirma: "la humanidad del hombre queda necesariamente expuesta a aquello que puede a un tiempo salvarla y aniquilarla” (Esposito, 2006, p. 11).

En este caso, el proceso de inmunización manifiesta una doble situación, pues aquellas técnicas que se aplican para proteger la vida, al mismo tiempo la niegan. Para el sostenimiento de una comunidad sana, se establecen una serie de técnicas que la hacen inmune a lo que la puede contaminar, no obstante, estos procesos inmunitarios crean fronteras que determinan qué es contrario a la comunidad y, por lo tanto, hay que prevenirla. Fenómenos políticos que se promulgan en nombre de la seguridad y el orden nacional revelan esta paradoja, pues debido a la protección de una vida nacional reconocida, personas que no pertenecen ni cumplen con los criterios nacionales, quedan expuestas y vulnerables a las decisiones políticas. Espósito evidencia cómo ese ejercicio biopolítico, ligado a los marcos jurídicos, van más allá, debido a que políticas que abanderan la protección de la vida, pueden llegar a excluirla.

En América Latina, Sonia Álvarez (2002, 2005 y 2008) en los estudios que hace acerca de los efectos del discurso del desarrollo en la región, particularmente en la consideración de la pobreza, analiza el surgimiento de nuevas formas de gobierno, en donde ya no importa que las poblaciones estén al servicio de la producción, sino el punto es neutralizar y controlar a las personas que el sistema político y económico han excluido, a partir de políticas sociales asistenciales (estas políticas son aquellas formas de obligación que se establecen en la modernidad para configurar las formas de reciprocidad entre el gobierno y los otros). Esta manera de ver las nuevas dinámicas de gobierno, la denomina la autora como focopolítica, bajo esta perspectiva, se empiezan a identificar las condiciones mínimas que requiere la existencia ("necesidades básicas"), para que las personas pobres, que no las pueden resolver bajo relaciones mercantiles, las puedan satisfacer a través de la promoción de un tipo de agencia filantrópica y solidaridad. Incluso se puede llegar a promover la identidad cultural para disminuir la discriminación, mientras las relaciones de inferioridad continúan. 
La idea de enfocarse en las "necesidades básicas", especialmente en la identificación de lo mínimo al margen de los deseos, fue una iniciativa promovida por los académicos del desarrollo, "neoclásicos de la idea de bienestar" y las organizaciones internacionales, como el Banco Mundial, el Fondo Monetario Internacional, junto con la promoción de organizaciones no gubernamentales; con el fin de intervenir la pobreza. Estos agentes comenzaron a promover una serie de políticas sociales, para que las personas pobres logren la satisfacción de sus necesidades mínimas, dejando poca responsabilidad al Estado. Los discursos del desarrollo social y el desarrollo humano promueven el acceso a mínimos biológicos, paquetes básicos de atención primaria en salud, educación, saneamiento básico. En este caso, se potencia un tipo de agencia, que permita que las personas se autogestionen lo mínimo, y que puedan convertir los activos escasos en satisfactorios (Álvarez, 2005).

Esta perspectiva: "neoclásicos de la idea de bienestar", considera que las necesidades básicas se pueden fijar y clasificar, al menos a un nivel mínimo de satisfacción. Parten del supuesto que las personas determinan sus preferencias a partir de la maximización individual. En este sentido, la pobreza no es un problema de distribución de recursos, sino del modo como las personas utilizan los recursos para satisfacer sus preferencias; por tanto, las personas pobres, clasificadas por un examen exógeno, solo podrían ocuparse de sus necesidades mínimas (Álvarez, 2005). Dentro de las posturas que cita la autora se encuentran los aportes que realizó Amartya Sen sobre la agencia y su influencia en la consideración sobre el desarrollo humano y la perspectiva de la “escala humana” de Max-Neef, Elizalde y Hopenhayn.

Bajo estas perspectivas, ¿en qué radicarían y cómo comprender las estrategias biopolíticas utilizadas para organizar el contexto rural colombiano?

\section{Incursión biopolítica de la vida campesina}

Los efectos biopolíticos para la población campesina en los años 90 se evidencian por lo menos en dos situaciones: a) a partir de la reproducción de representaciones simbólicas, provenientes de los modelos económicos de desarrollo que constituyen un sujeto desplazado, vulnerable, y que por lo tanto requiere de asistencia; b) en las medidas políticas que se tomaron para incentivar la producción en el campo y regular la tierra. A través del establecimiento de una agricultura comercial, que cambia de 
un mercado interno de sustitución de importaciones a un mercado internacional, en el que priman la exportación de bienes no transables.

\section{Representación simbólica del campesinado y biopolítica de las necesidades}

Hacer una reflexión en torno al sector campesino, en el contexto colombiano, requiere tener en cuenta, en primer lugar, la diversidad social y cultural, tanto de su población como de su territorio; en segundo lugar, tener presente las distintas transformaciones que sus organizaciones políticas y económicas han experimentado y logrado a lo largo de la historia; en tercer lugar, las categorías políticas, económicas y culturales con las que se han pensado sus problemas, especialmente sus necesidades; finalmente, las consecuencias que tiene la aplicación de estas categorías en la comprensión de la forma y el contenido de las dinámicas que revisten los territorios y su población.

Uno de los aspectos que se debe rastrear es el modo como en Colombia se fueron estableciendo y usando las categorías de "pequeño productor" y "trabajador agrario", especialmente en el manejo y sentido que le dieron las instituciones en el discurso político y económico. La Constitución Política en sus artículos: 64, 65 y 66 , da por sentado que ese es el modo como serán considerados los campesinos en la participación política, económica y social, sin embargo, ¿quién es un "trabajador agrario” en la Constitución Política? La carta política también sugiere para los "trabajadores agrarios" acceso a tierras, a servicios como salud, educación, vivienda, crédito y comercialización de productos para mejorar su calidad de vida, pero ¿qué calidad de vida les sugiere?

Haciendo un rastreo inicial, las categorías de "trabajador agrario" y "pequeño productor" responden al modo como en Colombia se ha tratado el tema de la propiedad de la tierra y la producción rural, ya que estos aspectos orientan la forma como se constituye la identidad y agencia de la población campesina. Existen tres perspectivas que se ocupan de un modo distinto de estos temas: la reforma agraria, la política de tierras, y el desarrollo rural. Tal y como lo menciona el informe, la política de reforma agraria y tierras en colombia del Centro Nacional de Memoria Histórica (CNMH, 2013), es importante considerar la distinción que hay entre estos tres enfoques, ya que se ocupan de una manera diferente de los aspectos mencionados. Por un lado, la reforma agraria, resalta la importancia de la redistribución de la 
propiedad de la tierra y los recursos naturales (Carrol, 1965; Dorner, 1972), junto con el cambio de la posición social y económica de los grupos sociales; por su parte, la política de tierras al manejo de los recursos, pero especialmente a las relaciones de poder que se configuran en la tenencia de la tierra; finalmente el desarrollo rural, al crecimiento de la producción, la productividad, el mejoramiento social y la formación de capital social. En Colombia, desde los años 70, la atención se enfocó en la última perspectiva, dejando de lado los problemas de la redistribución de la propiedad de la tierra y de los recursos, es por esa razón, que los cambios se encaminaron en empoderar a la sociedad civil, capacitar al campesinado, como factor de crecimiento y desarrollo. En este sentido, se hicieron esfuerzos por avanzar en el desarrollo rural y no en la redistribución de la tierra, por tanto, no se buscó modificar la posición de los campesinos como actor social, solo se siguió el afán de posicionarlo como un pequeño empresario.

En Colombia desde 1976 con el gobierno de Alfonzo López Michelsen, se comienzan a promover medidas de desarrollo rural, a través del Programa de Desarrollo Rural Integrado (DRI) que se desarrolló en tres fases, con el fin de responder a una estrategia de alimentación y nutrición para la población rural, concebida por el Banco Mundial y adoptada por los países del "tercer mundo", la idea era insertar en el sector: capital, tecnología y capacitación, a través de la revolución verde, con la idea de convertir a los pequeños agricultores en empresarios, pero solo a menor escala (Escobar, 2007), y así sacar del atraso al "tercer mundo".

Las tres fases del DRI se caracterizaron por: primera fase (1976-1981), de producción, que buscó establecer tecnologías apropiadas para desarrollar la economía campesina, junto con una intensificación del crédito, por medio de la Caja Agraria y programas sociales, en los que se incluyeron infraestructura, programas de educación y salud, para elevar el nivel de vida de la población rural. La segunda fase (1982-1989), hizo énfasis en la comercialización de los cultivos campesinos, en regiones de producción de pequeñas fincas. En esta época, los agricultores se vieron en la necesidad de comprar paquetes tecnológicos (uso de fertilizantes y semillas mejoradas), no obstante, con un alto costo. La tercera fase (1988-1993), el cambio tecnológico, concebido como la estrategia fundamental para el fortalecimiento de la producción.

El implemento del Programa de Desarrollo Rural Integrado (DRI), consistió en una serie de programas para aumentar la producción y la productividad de "pequeños agricultores", convirtiéndolos en agricultores comerciales a mediana escala, a partir de 
la adopción de capacitación, asistencia técnica y créditos. Los campesinos identificados como "malnutridos", "pequeños agricultores" y "agricultores sin tierra" los convirtió en clientes constituidos por el ojo del planificador, en nombre del progreso y el desarrollo. En este caso, el mecanismo regulador consistió en el establecimiento de un paquete tecnológico (crédito, asistencia técnica, semillas mejoradas, fertilizantes, control químico de plagas), para la especialización en ciertos cultivos, con el fin de homogenizar a la población rural, que de por sí es heterogénea. En este sentido, la formulación de diagnósticos y proyectos sociales de asistencia, con categorías que no tienen nada que ver con la comunidad a la que va dirigida, le crea y le impone una identidad, por medio de representaciones (Salgado, 2002) que caracterizan al campesinado como un ser fragmentado, débil, pobre, atrasado, incapaz para la participación y para el uso de los recursos, etc.

\footnotetext{
El imaginario del campesino pobre atrasado se enfrenta al del agente rural moderno y de espíritu empresarial, del modo que se opone la cultura campesina a la cultura moderna, la identidad campesina a la cultura moderna, la identidad campesina a la identidad occidental globalizada. (Salgado, 2002, p. 8)
}

Así los campesinos fueron modelados por una serie de técnicos y asesores, convirtiéndolos en "pequeños agricultores comerciales", modificando las relaciones cotidianas de los campesinos (Escobar, 2007). El problema, como lo veremos, es que el campesino es reducido solo como fuerza de trabajo, con ciertas capacidades que pueden ser incentivadas a favor de los mercados y de paso de la protección por parte de ellos, de sus necesidades básicas y derechos.

Es importante advertir que estas representaciones simbólicas tienen su justificación en el discurso del desarrollo que se configuró en América Latina, con el surgimiento de la pobreza como objeto de conocimiento, situación que implicó la constitución de instituciones, procesos socioeconómicos y factores tecnológicos, que tenían como finalidad combatirla (Escobar, 2007). Todo este aparataje se consolidó después de la Segunda Guerra Mundial, específicamente con el desplazamiento del discurso bélico al campo social, en países denominados como del "tercer mundo".

La categoría de desarrollo responde a un discurso en donde aparecen una serie de regímenes de representación que moldean la realidad y las acciones de las personas, en tanto que pone las reglas de juego acerca de "quién puede hablar, desde qué puntos 
de vista, con qué autoridad y según qué calificaciones, define las reglas a seguir para el surgimiento, denominación, análisis y eventual transformación de cualquier problema, teoría u objeto en un plan o política" (Escobar, 2007, p. 80).

Los regímenes de representación, para Escobar, son principios teóricos y metodológicos que permiten examinar los mecanismos y las consecuencias de la construcción del "tercer mundo", en últimas, representan un intento de trazar las cartografías o mapas de las configuraciones del saber y del poder que definen el periodo posterior a la Segunda Guerra. Regímenes de representación tales como: la pobreza y el subdesarrollo, impulsaron la construcción sistemática sobre el tercer mundo, junto con la implementación de ideas relacionadas con el desarrollo rural y el desarrollo sostenible. Detrás de esa buena intención, lo que comenzó a operar fue una serie de mecanismos sutiles, que tenían la intención de controlar y organizar a la población, dado que se convirtieron en blanco para "prácticas cada vez más sofisticadas y de una multiplicidad de programas aparentemente ineludibles" (Escobar, 2007, p. 77).

Como lo indica Escobar (2007), los regímenes de representación jugaron un papel importante en la implementación de los modelos de desarrollo en América Latina, pues a través de ellos es posible reconocer las posiciones que empiezan a ocupar las personas en las relaciones de poder. Uno de los efectos biopolíticos que han tenido esta representación negativa, radica en la modificación y control de sus necesidades, para el caso se retoma lo que Agnes Heller (1978) entiende por el concepto, quien asegura que las necesidades responden a una construcción histórica y a intereses que se imponen y circulan en las prácticas sociales a la que pertenecen los sujetos. Afirma que las instituciones imponen una serie de necesidades por medio de un discurso hegemónico, que en algunas ocasiones no tienen nada que ver con los sujetos a los que refieren. Este tipo de necesidades responden a necesidades manipuladas, que contrarias a otro tipo de necesidades (Heller, 1978) asegura la existencia de necesidades cualitativamente valiosas que le permiten al ser humano desarrollarse como especie, configurándose en contextos en donde la enajenación de la riqueza, la inversión de medios y fines y los seres humanos se convierten en un simple medio para la satisfacción de intereses privados de otro individuo.

El trabajo capitalista expresa este aspecto, ya que en la producción de mercancías, el valor de uso no sirve para la satisfacción de necesidades de quien con su trabajo produce el objeto, sino para la satisfacción de las necesidades de quien posee los medios de producción. Esta situación genera, según Heller, una homogenización de 
las necesidades, debido a que responden a un único interés. La necesidad humana hace parte de uno de los elementos, no el único, que posibilita las acciones humanas, sus capacidades y su identidad, debido que a través de ellas se resaltan una serie de intereses que permiten la existencia de seres heterogéneos. Para el caso de los campesinos, las necesidades son un elemento reivindicativo y emancipatorio de su modo de ser (Salgado y Prada, 2000); sin embargo, este aspecto distintivo suele ser vulnerable en el momento en el que otros grupos sociales o las mismas instituciones intentan imponer una serie de necesidades que tienden a homogeneizar y configurar su identidad.

La imposición de las necesidades se hace más fácil a partir de la construcción simbólica de la identidad, pues si tener en cuenta a las personas que dicen considerar, hace posible la imposición de necesidades externas. En este caso, se legitiman discursos discriminatorios y opresivos, más en un contexto en donde los procesos de subjetivación se hacen a partir de sistemas jurídicos que producen la identidad, para el sujeto que luego quieren representar desde un punto de vista político. En este caso la subjetividad es construida, establecida desde instancias dominantes que no tienen en cuenta los individuos a los que dicen referirse. Butler (2007) citando a Foucault, asegura:

Las nociones jurídicas de poder parecen regular la espera política en términos negativos, es decir, mediante la limitación, la prohibición, la reglamentación, el control y hasta la protección de las personas vinculadas a esa estructura política a través de la operación contingente y retractable de la elección. (p. 47)

Los procesos de reconocimiento y la representación política se hacen ignorando el contexto y las realidades que los individuos poseen y experimentan, situación que causa limitación, prohibición, etc.

La configuración de las necesidades, bajo el discurso del desarrollo, aniquila la heterogeneidad de la población campesina, es decir, de las distintas formas de vida, de creer, de sentir, sino que incentiva el establecimiento de un agente multifuncional empresario y competitivo, que ya no ve al campo como proveedor de alimentos y la necesidad de un modo de ser particular para producirlos, sino que ve al campo como una oportunidad de negocio, de turismo y artesanía (Méndez, 2013). En este sentido la agencia requiere un modo de ser particular con actitudes nuevas para el progreso. 
El campesinado es un sujeto histórico e intercultural, que constituye su modo de ser a partir de las relaciones que establece con la naturaleza, los ecosistemas, la tierra, con los oficios particulares que ejerce: tradicional, artesanal o moderno, en sectores como: la pesca, la siembra, etc., por el tipo y la diversidad en la producción, por la interacción con los recursos naturales y sociales, y finalmente, por las relaciones que establece con otros actores sociales - pobladores rurales, organizaciones públicas o privadas, entre otros-. Estos elementos de alguna manera caracterizan al "campesino ribereño", "campesino sabanero", "campesino cienaguero", "campesino costeño", "campesino friano o calentano".

La promoción de las "necesidades básicas" y el desarrollo de capacidades, particularmente en las políticas sociales que se refieren al campesinado colombiano en los años 90, hace que se configure un tipo de agencia particular con emociones que eliminen un comportamiento arcaico. En este caso un modo de ser con actitudes y emociones que no respondan a una cultura "arcaica" o "subdesarrollada", ya que estas son causantes de atraso y pobreza. Álvarez (2005) muestra cómo en América Latina con la entrada de la modernidad y con ella el discurso del desarrollo, las heterogeneidades de su población junto con sus prácticas fueron interpretadas como primitivas y causantes de la pobreza. Dado este aspecto, las categorías de pequeño productor y trabajador agrario responden a ese interés por homogenizar a una población que solo debe autogestionarse lo mínimo, con la limitación de lo que debe producir y someterse a la producción de una semilla certificada.

En síntesis, teniendo en cuenta la perspectiva biopolítica y la configuración de las necesidades de la población campesina, en los años 90 se consolida en las siguientes necesidades:

a) La creencia que solo bajo el saber científico y tecnológico es viable para el establecimiento de la calidad de vida de los campesinos.

b) Teniendo en cuenta las consecuencias de marginación que genera la confluencia, entre los marcos jurídicos y el modelo biopolítico. Esta situación que se da en el momento en el que la institucionalidad establece una serie de criterios, para la garantía de la población de ciertos beneficios, en el caso de la población campesina y de sus necesidades básicas. El punto es que bajo estos criterios se construye qué tipo de modo de vida se quiere reconocer. 
c) La necesidad de cambiar las formas de producción agrícola a una forma de producción industrial, que implica una amplia dependencia al mercado. Esto se ve en el fortalecimiento de las alianzas productivas, que ven como necesaria la asistencia del sector privado para el aprovechamiento de la tierra adjudicada a campesinos de escasos recursos, no obstante, lo que se dice es que el campesino, con la economía y la técnica familiar, no aprovecha adecuadamente la tierra. La carencia de infraestructura, condiciones geográficas y climáticas adversas, hace que se piense que los campesinos deban asociarse con empresarios que les permitan una eficacia en la producción.

\section{Forma de producción rural y biopolítica}

La subjetividad campesina responde a los efectos que han tenido las políticas neoliberales, este último entendido como un proyecto económico-político que tiene como fin establecer una estabilidad macroeconómica que permite el libre comercio, a partir de la desregulación del mercado y derechos de propiedad (Puello, 2015; Kay, 2016). No obstante, el discurso que no solo está orientado a elaborar reformas económicas y favorecer inversión extranjera, sino que es un proyecto político más amplio, que se caracteriza por afirmar los derechos humanos de los grupos minoritarios, para que entren al mercado económico (Hale, 2002). En los años 90 el discurso neoliberal intensifica la preocupación por competir de un modo eficaz en los mercados; no obstante, los resultados fueron nefastos, en tanto que no resolvió los problemas estructurales que presentaba el campo colombiano (Machado, 1998, p. 22). Estos aspectos han tenido como consecuencia la concentración de los recursos naturales y la precarización del trabajo rural (Kay, 2016).

Con la nueva Constitución se da paso a la apertura económica y con ella a la producción masiva de leyes que respondieron a las necesidades del mercado internacional (Peña, 2014). Este proceso fue denominado "la revolución pacífica" que en nombre de "la modernización" y "la protección ambiental", fomentó la producción de una "agricultura capitalista”, que con el apoyo de préstamos masivos de organizaciones internacionales como: El Fondo Monetario Internacional (FMI) y El Banco Mundial $(\mathrm{BM})$, estas organizaciones internacionales incentivaron el establecimiento de marcos normativos que obligaron a la formalización/consolidación de derechos de propiedad en el campo, para América Latina, con el fin de la apertura a la inversión extrajera (Garay, 2013, p. 167). Con el apoyo del Banco Mundial (BN), desde los 
años 60 los gobiernos de América Latina empezaron a darle un papel preponderante a la formulación de políticas transnacionales, con el fin de comercializar la tierra y dar paso a una reforma agraria asistida por el mercado (Veltmeyer, 2008; Pereria, 2016; Fajardo, 2009). Las políticas del BN denominadas políticas para la agricultura y desenvolvimiento rural (ADR) tenían como objetivo combatir la pobreza, a partir del libre comercio de tierras. Esta situación generó un cambio, a diferencia de los proyectos pasados, basados en una reforma agraria asistida por el Estado excesivamente centralizada, en donde este se veía como proveedor de crédito y servicio. El nuevo paradigma implantado en los 90 establece un nuevo tipo de relaciones público-privadas, en ese caso, asociaciones entre entidades, empresas y ONG para gobernar estos nuevos proyectos. Desde la década de los 80 ha aumentado el papel de las ONG, encargadas ahora de proyectos encaminados a solucionar conflictos ambientales y sociales (Pereira, 2016).

Desde una perspectiva biopolítica, es importante resaltar el papel que empiezan a jugar las ONG, pues con la pérdida de la participación del Estado en la formulación de políticas que permiten la garantía de los derechos sociales y económicos, las ONG son las que se apropian de la tarea, asumiendo una forma de "solidaridad particular", en donde los individuos son asistidos por ellos. Esta situación tiene efectos en la participación política de estas personas. En este caso, las necesidades empiezan a ser gestionadas por la intervención de nuevos agentes: ONG y empresas privadas. Esta situación se relaciona con las medidas que empezaron a implementarse con el fin de combatir la pobreza, por medio de medidas focalizadas, en donde el Estado pierde su papel protagonista e incentiva formas de "empoderamiento", en donde el individuo es el responsable de su situación. En este caso, estos agentes nuevos comienzan a fomentar maneras de "autosostenibilidad" y alianzas con las empresas privadas, sustituyendo la obligación que tiene el Estado de garantizar los derechos sociales (Álvarez, 2002).

Álvarez (2002) asegura que en esta época:

No es objetivo prioritario controlar los cuerpos y las vidas para obtener una mayor productividad. Se trata de territorializar y de producir formas de autocontrol comunitario sobre las poblaciones pobres, aunque se mantienen algunas de las técnicas de la biopolítica. (p. 59) 
Esta perspectiva, quiere aprovechar el "capital social” que tal y como lo entiende Bourdieu (1980), refiere al conjunto de recursos actuales o potenciales que sobrevienen de las capacidades y las relaciones que establecen los individuos en las comunidades a las que pertenecen. Este concepto se convirtió en un elemento fundamental para el BN, que remiten a las "redes en capitales de supervivencia”, es decir, a formas de organización de "autogestión” que han establecido las comunidades. Para resolver los conflictos sociales "el objetivo fue autonomizar a las poblaciones del Estado y a compensar su débil, escasa o nula relación con el mercado, así como fortalecer sus capacidades de autosubsistencia y de autogestión” (Álvarez, 2002, p. 74).

Bajo este panorama, el modelo agrario de los años 90 ha fortalecido la producción agroindustrial en detrimento de la producción campesina al cambiar la política de sustitución de importaciones, que en un primer momento ve al campesino como un individuo funcional, pero que con el advenimiento de las políticas neoliberales deja de serlo, para convertirse en un sujeto "explotado y excluido" (Tobasura, 2009). En un primer momento se consideró funcional, porque desde una visión del desarrollo sostenible, se necesitaba la formación de capital humano y la capacitación de agremiaciones dispuestas a la implementación de tecnología intensiva, para la exportación de productos agropecuarios. Con la implementación de las políticas neoliberales, el concepto de campesinos prósperos se evapora, debido a que unos pocos se convirtieron en asalariados de las nuevas empresas exportadoras, que no satisfacen los requerimientos mínimos laborales, como las famosas cooperativas de trabajo tan cuestionadas hoy, y otros, en una situación de desplazamiento. Estos aspectos en Colombia tuvieron los siguientes efectos: descenso de la inversión pública, desaparición de cultivos y concentración de la tierra, la agricultura parcelaria se vuelve improductiva, por la subutilización de los suelos y la mano de obra excedente (Fajardo, 2009).

Las normas jurídicas establecidas en el agro en Colombia están relacionadas con la regulación de los derechos de propiedad que han acrecentado la concentración y el despojo de la tierra, ya que están establecidas bajo una Constitución que no consideró a los campesinos como sujetos de políticas públicas y que no reguló frente a terrenos baldíos (Peña, 2014). Esto favorece a otros actores -elites locales- utilizando los procedimientos como el derecho real de superficie. Garay (2013) explica que el derecho real superficie, a pesar de que tiene su inicio en el 2005, comienza tiempo atrás, y consiste en un mecanismo de arrendamiento de uso del suelo, que facilita la titularización y "securitización” que beneficia la creación de un mercado del suelo. 
Estas medidas se justifican en libro II del Código Cívil colombiano. Aquí no se hace una reforma agraria, sino una política de tierras.

Esto quiere decir que el problema de la asignación de la tierra en Colombia no radica en su aplicación, sino a unos aspectos estructurales del derecho, que deja a la deriva y en una zona de indiferencia a los campesinos, ya que al no considerarlos, los ubica arbitrariamente en una condición de desplazamiento forzado y pobreza, tal y como lo indica el Tercer Censo Nacional Agropecuario (CNA) realizado por el DANE en el 2014 -desde 1970 no se realizaba uno-, donde asegura que la pobreza en el campo afecta al 44,7 \% de la población campesina, afectando también a 7,1 millones de hectáreas de cultivos; sin embargo, el 64,6 \% del área sembrada está destinada para la agroindustria.

Por otro lado, la Ley 160 de 1994 que consolida la empresarización del campo (Tobasura, 2009), sigue con una lógica de compra y venta de tierras, con poca posibilidad que los campesinos puedan acceder a la misma tierra, con el fin de acceder a nuevos propietarios rurales. En tanto que, a través del crédito, abre la posibilidad al sector financiero como administradores de los recursos. Entre los agentes involucrados en el mercado de tierras se encuentran (artículo 7): campesinos de bajos recursos, sujetos a reforma agraria, sociedades inmobiliarias rurales y personas naturales o jurídicas, estas últimas que ayuden al desarrollo. El INCORA, representante de la institucionalidad, simplemente fue mediador entre los agentes. La carencia de infraestructura, condiciones geográficas y climáticas adversas, hace que se piense que los campesinos deban asociarse con empresarios, permitiéndoles una mayor eficacia en la producción. En este sentido, su agencia es establecida por un tipo de solidaridad con el sector privado, a través de alianzas productivas. Estas asociaciones, como las ha documentado Alfredo Molano en su texto Dignidad campesina, consisten en ofrecer la tierra para el sector empresarial y abrir campo al monocultivo, esto tuvo como consecuencia la expansión de tierras que eran comunales y el endeudamiento por parte de la población, ya que esta comenzó a respaldar los créditos con tierra (Molano, 2013).

Esta ley considera que el sector rural necesita inversión privada, por esta razón establece que través de compra de tecnología de punta y por medio de asociaciones productivas, entre la empresa y los campesinos, la población y el campesino lograrán la calidad de vida que requiere. Las zonas de desarrollo empresarial tienen aún mayor relevancia, mientras las zonas de reserva campesina fueron estigmatizadas y poco 
recocidas. Por un lado, las zonas de desarrollo empresarial (ZDE), reguladas con el Acuerdo 028 de diciembre de 1995 de la Junta Directiva del INCORA, tiene el objetivo de proteger y promover la inversión de capital privado en terrenos baldíos. En este caso, las normas que acompañan a esta ley establecen una igualdad formal entre campesinos y los representantes del sector empresarial, generando una competencia desigual. Esta situación es problemática, porque fortalece la proletarización campesina. Por otro, las zonas de reserva campesina (ZRC), zonas de colonización, que provienen de la lucha de estas comunidades, refieren a lugares que tienen como fin proteger la economía campesina.

Del mismo modo, en esta época comienza la comercialización de bienes y servicios ambientales, en tanto que se empiezan a considerar como mercancías que permiten una acumulación nueva de capital, entre estos bienes se encuentran: el aprovechamiento del agua para el riego y la producción agroindustrial, materias primas para la generación de energía, investigación de la biodiversidad para la comercialización del saber tradicional y comercialización de semillas mejoradas. De alguna manera, estas construcciones intentan legitimar unas formas de saber que comienzan a cobrar importancia, ya que en nombre de la seguridad alimentaria se empiezan a consolidar compañías biotecnológicas (Monsanto, Syngenta, Dow Agrosciences, DuPont, Bayer y BASF), que bajo la ampliación de los derechos de propiedad, controlan el uso tradicional de la semilla, modificando así las formas de vida que giran en torno a este tipo de siembra, junto con la autonomía de las personas. En Colombia, por ejemplo, bajo la decisión 345 de 1993 de la Comisión Comunidad Andina (CAN), se crea el "régimen común de protección a los derechos de los obtentores de variedades vegetales", con el fin de reglamentar y patentar las variedades de vegetales y la manipulación genética. Esta situación genera las condiciones para la monopolización de las semillas y el acaparamiento del saber que se ha construido alrededor de ellas (Gutiérrez, 2015), favoreciendo a la agricultura industrial y al fortalecimiento de alianzas productivas entre el campesino y el sector privado, consolidadas en el 2002 y que, paradójicamente, han incrementado las situaciones de desigualdad en la producción y el consumo de alimentos (Grupo Semillas, 2011).

El reconocimiento de los campesinos es precario, debido a que este se da por medio de una identidad impuesta, configurada por una serie de representaciones simbólicas negativas, que facilita desde una perspectiva biopolítica el control y gestión de sus necesidades, en un contexto en donde el ejercicio soberano es débil y reina una 
soberanía en vilo. El territorio colombiano es tan amplio que ciertos lugares quedan fuera del control institucional (González, 1989; Uribe, 1999) dando como consecuencias situaciones de discriminación y marginalización de la vida, que no tiene que ver con un exceso de poder, sino por un déficit de este. Allí la ciudadanía se pone en cuestión, "cuando la soberanía permanece en vilo, en arcos de tiempo prolongados, la ciudadanía se torna virtual y los derechos de todos se hacen precarios y vulnerables" (Uribe, 1999, p. 30). Es importante advertir que, aun cuando el ejercicio del poder soberano revista un carácter distinto en nuestro contexto, las consecuencias suelen evidenciar el resultado de decisiones biopolíticas, ya que tienen como efecto el incremento de la marginalización, en este caso de la vida campesina, causado por un "apartheid institucional", que según García y Espinosa (2013), responden a la precariedad del Estado, dado que los derechos de las personas no son reconocidos, ni protegidos. Esta situación facilita el aumento de conflictos rurales, la producción de actividades ilícitas, el incremento de la pobreza y el desplazamiento forzado (Uribe, 2000), junto con la destrucción de los recursos naturales (PNUD, 2011).

Es importante recalcar, que este aspecto, no sería posible sin la construcción imaginaria de un campesinado "atrasado", ya que a partir de esta idea se comienza a adjudicar un paquete de necesidades que tienen el objetivo de sacar al campesino de su incapacidad y atraso. El presupuesto que atraviesan los marcos normativos políticos y económicos que tienen que ver con el sector rural, en esta época, refieren a una definición moderna de desarrollo que se caracteriza por proponer una visión que no responde necesariamente a las circunstancias y posibilidades en las que se encuentra el sector rural del momento, sino a una manera como se quiere que la ruralidad se organice y funcione. Teniendo en cuenta la realidad que revela el contexto colombiano, es importante conocer el papel que juegan las representaciones simbólicas, tanto en la configuración del espacio como de las personas que las ocupan, pues estás revelan la manera como el Estado forja la alteridad. Podemos decir que los marcos normativos, según las representaciones simbólicas con los que estén formulados, pueden generar las condiciones de posibilidad para que un sujeto pueda aumentar sus opciones de acción y de agencia política, sin embargo, tal capacidad depende de las relaciones de poder y de las estrategias biopolíticas que se entretejen en el ejercicio político. 


\section{Referencias}

Álvarez, S. (2008). Pobreza y desarrollo en América Latina. Salta: Universidad de Salta.

Álvarez, S. (2005). Los discursos minimistas sobre las necesidades básicas y los umbrales de ciudadanía como reproductores de pobreza. En S. Álvarez (Comp.). Trabajo y producción de la pobreza en Latinoamérica y El Caribe: estructuras, discursos y actores, (pp. 239-273). Buenos Aires: Consejo Latinoamericano de Ciencias Sociales CLACSO.

Álvarez, S. (2002, enero-abril). La transformación de las instituciones de reciprocidad y control: del don al capital social y de la biopolítica a la focopolítica. Revista Venezolana de Economía y Ciencias Sociales, 8(1), 57-89.

Artículo 64. (1991). Es deber del Estado promover el acceso progresivo a la propiedad de la tierra; de los trabajadores agrarios, en forma individual o asociativa, y a los servicios de educación, salud, vivienda, seguridad social, recreación, crédito, comunicaciones, comercialización de los productos, asistencia técnica y empresarial, con el fin de mejorar el ingreso y calidad de vida de los campesinos. Bogotá, Colombia: Constitución Política de Colombia. Recuperado de http://www.constitucioncolombia.com/titulo-2/capitulo-2/articulo-64

Artículo 65. (1991). La producción de alimentos gozará de la especial protección del Estado. Para tal efecto, se otorgará prioridad al desarrollo integral de las actividades agrícolas, pecuarias, pesqueras, forestales y agroindustriales, así como también a la construcción de obras de infraestructura física y adecuación de tierras. De igual manera, el Estado promoverá la investigación y la transferencia de tecnología para la producción de alimentos y materias primas de origen agropecuario, con el propósito de incrementar la productividad. Bogotá, Colombia: Constitución Política de Colombia. Recuperado de http://www.constitucioncolombia.com/ titulo-2/capitulo-2/articulo-65

Artículo 66. (1991). Las disposiciones que se dicten en materia crediticia podrán reglamentar las condiciones especiales del crédito agropecuario, teniendo en cuenta los ciclos de las cosechas y de los precios, como también los riesgos inherentes a la actividad y las calamidades ambientales. Bogotá, Colombia: Constitución Política de Colombia. Recuperado de http://www.constitucioncolombia.com/titulo-2/ capitulo-2/articulo-66 
Bourdieu, P. (1980). Le Capital Social, actes de la recherche. Sciencies Sociales, 31, 3-6.

Carrol, F. (1965). La estructura agraria. Reformas agrarias en América Latina, procesos y perspectivas. México: Fondo de Cultura Económica.

Dorner, P. (1972). Reforma agraria y desarrollo económico. Madrid: Alianza.

Butler, J. (2007). El género en disputa. Barcelona: Paidós.

Centro Nacional de Memoria Histórica. (2013). La política de reforma agraria y tierras en Colombia.

Chaparro, A., y Galindo, C. (2009). Génesis y transformaciones del Estado Nación en Colombia. Una mirada topológica a los estudios sociales desde la filosofía política. Bogotá: Universidad del Rosario.

Departamento Administrativo Nacional de Estadística (DANE). (2014). Censo nacional Agropecuario 2014. Recuperado de http://www.dane.gov.co/index.php/ estadisticas-por-tema/agropecuario

DPN. (1991). La revolución pacífica. Plan de desarrollo económico y social 1990-1994. Bogotá.

DPN. (1998). Informe de desarrollo para Colombia 1998. TM Editores.

Escobar, A. (2007). La invención del tercer mundo: construcción y deconstrucción del desarrollo. Caracas: Fundación el perro y la rana.

Esposito, R. (2006). Bíos. Biopolítica y filosofía. Buenos Aires: Amorrortu Editores.

Fajardo, D. (2009). Territorios de agricultura colombiana. Bogotá: Universidad Externado de Colombia.

Forero, J. (2010). El campesinado colombiano: entre el protagonismo económico y el desconocimiento de la sociedad. Bogotá: Pontificia Universidad Javeriana.

Foucault, M. (1976). Vigilar y castigar. México: Siglo XXI Editores.

Foucault, M. (1998). Las palabras y las cosas. Una arqueología de las ciencias humanas. México: Siglo XXI Editores.

Foucault, M. (2000). Defender la sociedad. México: Fondo de Cultura Económica. 
Foucault, M. (2006). Seguridad, territorio, población. México: Fondo de Cultura Económica.

Foucault, M. (2007). Historia de la sexualidad I. México: Fondo de Cultura Económica.

García, M., y Espinosa, J. (2013). El derecho al Estado. Los efectos legales del apartheid institucional. Bogotá: Centro de Estudios de Derecho, Justicia y Sociedad.

Garay, L. J., et al. (2013). Reflexiones sobre la ruralidad y el territorio en Colombia. Problemática y retos actuales. Bogotá: Oxfam. Recuperado de http://www. congresoeducacionruralcoreducar.com/images/Doc_web/62.-Reflexiones-sobrela-ruralidad-y-el-territorio-en-Colombia.pdf

González, F. (2016). Lo político reconsiderado desde lo común. Cuadernos de Filosofía Latinoamericana, 37(114), 187-201.

González, F. (1989). Aproximación a la configuración política de Colombia. Controversia, (154). Bogotá: Cinep.

Gutiérrez, L. (2015). Soberanía alimentaria. La red de semillas libres de Colombia. [Con]Textos, 4(13), 11-24.

Grupo Semillas. (s.f.). Las leyes de semillas, aniquilan la soberanía y autonomía alimentaria de los pueblos. Bogotá: Grupo Semillas. Recuperado de http://www. semillas.org.co/apc-aa-files/5d99b14191c59782eab3da99d8f95126/Leyes_de_ semillas.Colombia.pdf

Guzman, N. (2016). Las rutas del viaje en la cuestión del reconocimiento: un diálogo con la obra de Judith Butler. Cuadernos de Filosofía Latinoamericana, 37(114), 133-148.

Hale, C. R. (2002). Does Multiculturalism Menace? Governance, Cultural Rights and The Politics of Identity in Guatemala. Journal of Latin American Studies, (34), 485-524. Cambridge University Press.

Heller, A. (1978). Teoría de las necesidades en Marx. Barcelona península.

Kay, C. (2016). La transformación neoriberal del mundo rural: procesos de concentración de la tierra y del capital y la intensificación de la precariedad del trabajo. Revista Latina Americana de Estudios Rurales - ReLaER, 1(1), 1-26. 
Krantz, L. (1977). El campesino como concepto analítico. Nueva Antropología. Revista de Ciencias Sociales, (6), 87-98.

Ley 160. (1994). Por la cual se crea el Sistema Nacional de Reforma Agraria y Desarrollo Rural Campesino, se establece un subsidio para la adquisición de tierras, se reforma el Instituto Colombiano de la Reforma Agraria y se dictan otras disposiciones. Diario Oficial n. ${ }^{\circ}$ 41.479. Bogotá. Recuperado de http://www.urosario.edu.co/ jurisprudencia/catedra-viva-intercultural/Documentos/ley_160_de_1994.htm

Machado. A. (1998). La cuestión agraria en Colombia a fines del milenio. Bogotá: El Áncora Editores.

Méndez, Y. (2013). Derecho a la tierra y al territorio, justicia y zonas de reserva campesina: El caso del Valle de Río de Cimitarra. (Tesis de maestría en Desarrollo Rural). Bogotá, Colombia: Pontifica Universidad Javeriana.

Molano, A. (2013). Dignidad campesina. Entre la realidad y la esperanza. Bógota: Códice.

Llambí, L. (1990). Procesos de transformación del campesinado latinoamericano. En F. Bernal (Comp.). El campesinado contemporáneo. Cambios recientes en los países andinos. Bogotá: Cerec, Tercer Mundo Editores.

Pereira, J. (2016, jan-abr). Modernização, combate à pobreza e mercado de terras Uma análise das políticas do Banco Mundial para agricultura e desenvolvimento rural (1944-2003). Varia Historia, Belo Horizonte, 32(58), 225-258.

Peña, R. P., Parada, M. M., y Zuleta, S. (2014). La regulación agraria en Colombia o el eterno déjà vu hacia la concentración y el despojo: un análisis de las normas jurídicas colombianas sobre el agro (1991-2010). Estudios Socio-Jurídicos, 16(1), 121-164.

PNUD. (2011). Colombia rural razones para la esperanza. Informe Nacional de Desarrollo Humano 2011. INDH PNUD.

Puello, J. (2015). Neoliberalismo, antineoliberalismo, nuevo neoliberalismo, episodios y trayectorias económico-políticas suramericanas (1973-2015). En L. Rojas (Coord.) Neoliberalismos en América Latina. Crisis, tendencias y alternativas. Asunción: CLACSO. 
Rincón, J. (2006). Movilización social y desarrollo rural en Colombia: del sistema agrícola a la nueva ruralidad. Revista Colombiana de Sociología, (27), 63-98.

Salgado, C. (2002). Los campesinos imaginados. Bogotá: Cuadernos Tierra y Justicia.

Salgado, C. (2014). Colombia: Estado actual del debate sobre el desarrollo rural. Bogotá: Desde Abajo.

Salgado, C., y Prada E. (2000). Campesinado y protesta social:1980-1995. Bogotá: CINEP.

Schmitt, C. (2014). El concepto de lo político. Madrid: Alianza.

Schmitt, C. (2012). Sobre los tres modos de pensar la Ciencia Jurídica. Madrid: Tecnos.

Tobasura, I. (2009, julio-diciembre). De campesinos a empresarios. La retórica neoliberal de la política agraria en Colombia. Revista Nera, 12(5), 7-21.

Uribe, M. T. (1999, julio-diciembre). Las soberanías en disputa: ¿Conflicto de identidades o de derechos? Estudios Políticos, (15). Medellín: Instituto de Estudios Políticos, Universidad de Antioquia.

Uribe, M. T. (2000, julio-diciembre). Notas para la conceptualización del desplazamiento forzado en Colombia. Estudios Políticos, (17), Medellín: Instituto de Estudios Políticos, Universidad de Antioquia.

Veltmeyer, H. (2008). La dinámica de las ocupaciones de tierras en América Latina. En M. Sam y Y. Paris (Coord.). Recuperando la tierra. El resurgimiento de movimientos rurales en África, Asia y América Latina. Buenos Aires: CLACSO, Consejo Latinoamericano de Ciencias Sociales. 